\title{
Inverse see-saw neutrino masses in the Littlest Higgs model with T-parity
}

\section{Francisco del Aguila, José Ignacio Illana, José María Pérez-Poyatos and José Santiago}

CAFPE and Departamento de Fúsica Teórica y del Cosmos,

Universidad de Granada, E-18071 Granada, Spain

E-mail: faguila@ugr.es, jillana@ugr.es, jmppoyatos@ugr.es, jsantiago@ugr.es

ABSTRACT: We show that the inverse see-saw is the most natural way of implementing neutrino masses in the Littlest Higgs model with T-parity. The three extra quasi-Dirac neutrinos are needed to cancel the quadratically divergent contributions of the mirror leptons to the Higgs mass. If the T-parity of the heavy neutrino singlets is chosen to be even, their contributions to lepton flavor violating transitions are one-loop finite. The most stringent limits on this scenario result from the non-observation of these transitions. Constraints on neutrino mixing imply an upper bound on the mass of the T-odd mirror leptons at the reach of the LHC and/or future colliders.

Keywords: Beyond Standard Model, Neutrino Physics

ARXIV EPRINT: 1910.09569 


\section{Contents}

1 Introduction 1

2 Neutrino masses in the LHT 2

2.1 T-odd heavy singlet 5

$\begin{array}{lll}2.2 & \text { See-saw of type I and II } & 7\end{array}$

3 Inverse see-saw masses and mixings $\quad 9$

3.1 LFV limits 9

4 Conclusions $\quad 12$

\section{Introduction}

After the discovery of the Higgs boson [1, 2], there are increasing indications of a mass gap to the next physics scale, $f$, above the $\mathrm{TeV}[3,4]$. Such a scenario can be naturally implemented in non-minimal supersymmetric models, as well as in composite Higgs models [5-7], with the scalar observed at the LHC being a pseudo-Nambu-Goldstone (NG) boson in this latter case. Among this second class of models, the Littlest Higgs model with T-parity (LHT) [8-12] emerges as a well-motivated and phenomenologically viable simple model [13-32].

As a matter of fact, neutrino physics remains the only signal of new physics beyond the minimal Standard Model (SM) [33]. Then, although the LHT is designed to interpret the Higgs boson as a pseudo-NG boson, it must also account for the observed neutrino masses and mixing. As we shall see, T-parity, which plays an essential phenomelogical role suppressing new indirect effects and reducing direct production limits of new (T-odd) particles for they must be pair-produced, also has a significant impact on the mechanism of neutrino mass generation.

In this paper we show that the LHT can naturally accommodate the inverse see-saw of type I [34-36], and the observed pattern of neutrino masses and mixing [37], without breaking T-parity. Lepton Number (LN) must be explicitly broken at some stage if the observed neutrinos acquire Majorana masses. The LHT has the matter content to account for see-saw mechanisms of type I and II [38-47]. However, the type II see-saw, originally considered in the literature [48], relies on the spontaneous breaking of T-parity [21]. Even more, the invoked coupling giving neutrinos a mass explicitly breaks T-parity, what implies that it can not be generated by quantum corrections as we argue below.

In the following we shall show that the minimal lepton content of the model is fixed if one requires that the Higgs mass does not receive quadratically divergent contributions and that Lepton Flavor Violating (LFV) processes, in particular, Higgs decays into two 
different opposite-charge leptons, remain one-loop finite. This is assuming the originally proposed mechanism for implementing T-parity in the fermion sector and the associated Yukawa Lagrangian giving masses to the T-odd partners of the SM fermion doublets (mirror fermions) [11, 12]. LFV processes stay one-loop finite when we also assume that the SM right-handed $(\mathrm{RH})$ charged leptons are singlets under the global symmetry and that they obtain masses through the usual (minimal) Yukawa interaction $[13,15,28]$. However, the finiteness of these processes is only guaranteed if we require the heavy neutrino singlets completing the RH multiplets under the unbroken global symmetry to be T-even. In this case they mix with SM neutrinos already at tree level and the corresponding mass matrix is the inverse see-saw one, once small Majorana masses are assumed for their heavy lefthanded (LH) singlet counterparts. Hence, all phenomenological implications derived from this mechanism follow, in particular, the constraints on the mixing between SM and heavy leptons obtained from Electro-Weak Precision Data (EWPD) and from the non-observation of LFV processes [49-52], as we shall summarize. The conclusion is that, even though Tparity alleviates the flavor problem, we still have to tune the model to reduce the possible misalignment between the SM and the heavy fermions in the absence of an extra flavor symmetry. Constraints on neutrino mixing result in an upper bound on the mass of T-odd mirror leptons, which are at the reach of the LHC and/or future colliders.

In next section we introduce the notation and justify why the inverse see-saw is naturally implemented in the LHT. In particular, we emphasize that the see-saw of type II must be expected to be suppressed relative to the see-saw of type I. In section 3 we review the current constraints on the inverse see-saw and the allowed regions of LHT parameters. The last section is devoted to conclusions and final comments on the implications for LHC searches.

\section{Neutrino masses in the LHT}

Let us introduce the LHT to fix our notation and assumptions [25, 31]. (For excellent reviews see [53-55].) The model realizes non-linearly the global SU(5) symmetry which is broken down to $\mathrm{SO}(5)$, giving rise to $14 \mathrm{NG}$ bosons

$$
\Pi=\left(\begin{array}{ccccc}
-\frac{\omega^{0}}{2}-\frac{\eta}{\sqrt{20}} & -\frac{\omega^{+}}{\sqrt{2}} & -i \frac{\pi^{+}}{\sqrt{2}} & -i \Phi^{++} & -i \frac{\Phi^{+}}{\sqrt{2}} \\
-\frac{\omega^{-}}{\sqrt{2}} & \frac{\omega^{0}}{2}-\frac{\eta}{\sqrt{20}} \frac{v+h+i \pi^{0}}{2} & -i \frac{\Phi^{+}}{\sqrt{2}} & \frac{-i \Phi^{0}+\Phi^{P}}{\sqrt{2}} \\
i \frac{\pi^{-}}{\sqrt{2}} & \frac{v+h-i \pi^{0}}{2} & \sqrt{\frac{4}{5} \eta} & -i \frac{\pi^{+}}{\sqrt{2}} & \frac{v+h+i \pi^{0}}{2} \\
i \Phi^{--} & i \frac{\Phi^{-}}{\sqrt{2}} & i \frac{\pi^{-}}{\sqrt{2}} & -\frac{\omega^{0}}{2}-\frac{\eta}{\sqrt{20}} & -\frac{\omega^{-}}{\sqrt{2}} \\
i \frac{\Phi^{-}}{\sqrt{2}} & \frac{i \Phi^{0}+\Phi^{P}}{\sqrt{2}} & \frac{v+h-i \pi^{0}}{2} & -\frac{\omega^{+}}{\sqrt{2}} & \frac{\omega^{0}}{2}-\frac{\eta}{\sqrt{20}}
\end{array}\right) .
$$

They act on the fundamental representation of the unbroken subgroup multiplying by $\xi=e^{i \Pi / f}$. The action of T-parity is defined to make T-odd all but the SM scalar doublet 
$\phi=\left(-i \pi^{+}\left(v+h+i \pi^{0}\right) / \sqrt{2}\right)^{T}$ of hypercharge $1 / 2(v \simeq 246 \mathrm{GeV}$ is the $\mathrm{SM}$ vacuum expectation value (vev) and the superscript $T$ means transpose):

$$
\Pi \stackrel{\mathrm{T}}{\longleftrightarrow}-\Omega \Pi \Omega, \quad \Omega=\operatorname{diag}(-1,-1,1,-1,-1) .
$$

Four of them, $\omega$ and $\eta$, are eaten by the T-odd replica of the electro-weak gauge bosons whereas the other six, $\Phi$, transform as a complex electro-weak triplet of hypercharge 1.

In the fermion sector each SM lepton doublet $l_{L}=\left(\nu_{L} \ell_{L}\right)^{T}$ is doubled introducing two incomplete quintuplets $[10,11]\left(\sigma^{2}\right.$ is the second Pauli matrix):

$$
\Psi_{1}=\left(\begin{array}{c}
-i \sigma^{2} l_{1 L} \\
0 \\
0
\end{array}\right), \quad \Psi_{2}=\left(\begin{array}{c}
0 \\
0 \\
-i \sigma^{2} l_{2 L}
\end{array}\right),
$$

with $\Psi_{2}$ transforming with the fundamental SU(5) representation $V$ and $\Psi_{1}$ with its complex conjugated $V^{*}$,

$$
\Psi_{1} \longrightarrow V^{*} \Psi_{1}, \quad \Psi_{2} \longrightarrow V \Psi_{2}
$$

The indices 1 and 2 must not be confused with the family index, which we will omit if not necessary. The action of T-parity on the LH leptons is then defined to be

$$
\Psi_{1} \stackrel{\mathrm{T}}{\longleftrightarrow} \Omega \Sigma_{0} \Psi_{2}, \quad \text { with } \quad \Sigma_{0}=\left(\begin{array}{ccc}
0 & 0 & \mathbf{1}_{2 \times 2} \\
0 & 1 & 0 \\
\mathbf{1}_{2 \times 2} & 0 & 0
\end{array}\right) \text {. }
$$

T-parity is thus implemented in the fermionic sector duplicating the SM doublet $l_{L}=$ $\left(l_{1 L}-l_{2 L}\right) / \sqrt{2}$, corresponding to the T-even combination $\left(\Psi_{1}+\Omega \Sigma_{0} \Psi_{2}\right) / \sqrt{2}$, with an extra heavy mirror doublet $l_{H L}=\left(\nu_{H L} \ell_{H L}\right)^{T}=\left(l_{1 L}+l_{2 L}\right) / \sqrt{2}$ obtained from the T-odd orthogonal combination $\left(\Psi_{1}-\Omega \Sigma_{0} \Psi_{2}\right) / \sqrt{2}$. This extra doublet per family will get its mass combining with a $\mathrm{RH}$ doublet $l_{H R}$ in an $\mathrm{SO}(5)$ multiplet $\Psi_{R}$, transforming with the fundamental $\mathrm{SO}(5)$ representation $U$,

$$
\Psi_{R}=\left(\begin{array}{c}
\psi_{R}^{\prime} \\
\chi_{R} \\
-i \sigma^{2} l_{H R}
\end{array}\right), \quad \Psi_{R} \longrightarrow U \Psi_{R} .
$$

The non-linear Yukawa coupling generating this large mass $\sim f$ reads

$$
\mathcal{L}_{Y_{H}}=-\kappa f\left(\bar{\Psi}_{2} \xi+\bar{\Psi}_{1} \Sigma_{0} \xi^{\dagger}\right) \Psi_{R}+\text { h.c. },
$$

where the first term preserves the global symmetry for $\xi \rightarrow V \xi U^{\dagger}$. While the second one is its T-transformed once the T-transformed of $\Psi_{R}$ is fixed to be $\Omega \Psi_{R}[12,56,57]$.

This Yukawa Lagrangian then constrains the heavy fermion content, also restricting the see-saw pattern, as we discuss in the following. Besides giving a vector-like mass $\sqrt{2} \kappa f$ to $\nu_{H}$, it also gives a quadratically divergent contribution to the Higgs mass through the 

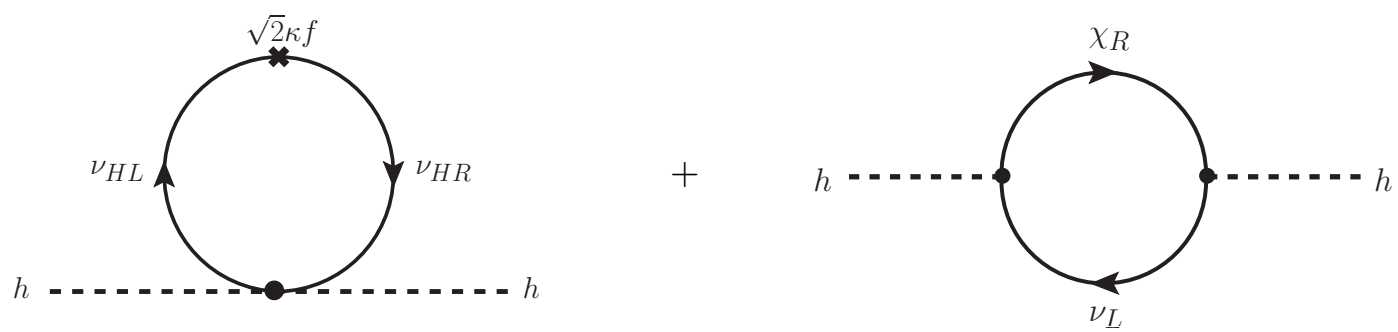

Figure 1. Diagrams contributing to the Higgs mass. The mirror lepton exchange (left) cancels the mirror-singlet mixing contribution (right).

diagram in figure 1 (left). This contribution with a mass insertion and $\nu_{H L, R}$ running in the loop is cancelled by the contribution of the diagram in figure 1 (right) with $\chi_{R}$ and $\nu_{L}$ running in the loop. This cancellation is exact if the masses of $\chi$ and $\nu_{H}$ are equal. Otherwise, the sum of both contributions is logarithmically divergent (see, for instance, $[58,59]$ for the analogous cancellation in the collective breaking case). Hence, $\chi_{R}$ can not be ignored [11]. ${ }^{1}$ Few comments are in order. The T-parity of $\chi_{R}$ is even in contrast with the other four components in $\Psi_{R}$, which are odd. If we had chosen the T-transformed of $\Psi_{R}$ to be $-\Psi_{R}$ and the T-parity of $\chi_{R}$ to be also odd, the lepton running in the diagram of figure 1 (right) would have been $\chi_{R}$ and $\nu_{H L}$, and not $\nu_{L}$. Obviously, including all field components both in the $\mathrm{LH} \mathrm{SU}(5)$ and the $\mathrm{RH} \mathrm{SO}(5)$ multiplets, the total contribution to the Higgs mass cancels due to the NG nature of the Higgs boson. However, as we want only to duplicate the SM (LH) lepton doublets to start with, the SM singlet $\chi_{R}$ must be always included to cancel the quadratically divergent contribution of $\nu_{H}$ to the Higgs mass. This can be checked diagramatically working out the corresponding Feynman rules for the Lagrangian in eq. (2.7) and computing the diagrams in figure 1, or reading the Higgs mass from the general Coleman-Weinberg expression [8, 60, 61]

$$
\mathcal{V}^{1-\text { loop }}=\frac{1}{32 \pi^{2}} \operatorname{Str}\left(\mathcal{M}^{2}\right) \Lambda^{2}+\frac{1}{64 \pi^{2}} \operatorname{Str}\left(\mathcal{M}^{4}\right) \ln \frac{\operatorname{Str}\left(\mathcal{M}^{2}\right)}{\Lambda^{2}}+\ldots
$$

where $\operatorname{Str}\left(\mathcal{M}^{n}\right)=\sum_{p}(-1)^{2 s_{p}}\left(2 s_{p}+1\right) m_{p}^{n}$ runs over all particles with spin $s_{p}$ and background dependent mass $m_{p}$ and $\Lambda$ is the momentum cut-off $\sim 4 \pi f .{ }^{2}$

The lepton singlets $\chi_{R}$ must also get a large (vector-like) mass by combining with a LH singlet $\chi_{L}$ through a direct mass term without further couplings to the Higgs. As they must do the extra leptons (partner doublets) in $\mathrm{SO}(5)$ multiplets, $\psi_{R}^{\prime}$ in eq. (2.6), for they must be also included in order to keep the LFV Higgs decay amplitudes into charged leptons one-loop finite [31]. (See also footnote 1.) Thus, their mass terms write

$$
\mathcal{L}_{M}=-M \overline{\chi_{L}} \chi_{R}-M^{\prime} \overline{\psi_{L}^{\prime}} \psi_{R}^{\prime}+\text { h.c. }
$$

\footnotetext{
${ }^{1}$ Incomplete $\mathrm{SO}(5)$ fermion representations also result in two-loop quartically divergent contributions to the Higgs mass induced by the couplings of their kinetic term [10].

${ }^{2}$ Note that for fermions $\mathcal{M}^{2} \equiv \mathcal{M} \mathcal{M}^{\dagger}$.
} 
where $\psi_{L}^{\prime}$ is the LH (doublet) counterpart of $\psi_{R}^{\prime}{ }^{3}$ (Nevertheless, in the following we will not be concerned with the mirror leptons or their partners $\psi^{\prime}$ because they are T-odd and do not mix with the SM leptons nor with $\chi$.) With this matter content, $\chi_{L}$ is an $\operatorname{SU}(5)$ singlet and it is therefore natural to include a small Majorana mass for it. Once LN is assumed to be only broken by small Majorana masses $\mu$ in the heavy LH neutral sector,

$$
\mathcal{L}_{\mu}=-\frac{\mu}{2} \overline{\chi_{L}^{c}} \chi_{L}+\text { h.c. },
$$

the resulting (T-even) neutrino mass matrix reduces to the inverse see-saw one:

$$
\mathcal{L}_{M}^{\nu}=-\frac{1}{2}\left(\overline{\nu_{L}^{c}} \overline{\chi_{R}} \overline{\chi_{L}^{c}}\right) \mathcal{M}_{\nu}^{T-\text { even }}\left(\begin{array}{c}
\nu_{L} \\
\chi_{R}^{c} \\
\chi_{L}
\end{array}\right)+\text { h.c. },
$$

where

$$
\mathcal{M}_{\nu}^{T-\text { even }}=\left(\begin{array}{ccc}
0 & i \kappa^{*} f \sin \left(\frac{v}{\sqrt{2} f}\right) & 0 \\
i \kappa^{\dagger} f \sin \left(\frac{v}{\sqrt{2} f}\right) & 0 & M^{\dagger} \\
0 & M^{*} & \mu
\end{array}\right),
$$

with each entry standing for a $3 \times 3$ matrix to take into account the 3 lepton families. The $\kappa$ entries are given by the Yukawa Lagrangian in eq. (2.7) and $M$ stands for the direct heavy Dirac mass matrix in eq. (2.9), while $\mu$ is the mass matrix of small Majorana masses in eq. (2.10). The natural size of the mass eigenvalues for $M$ is $\sim 10 \mathrm{TeV}$, of the order of $4 \pi f$ with $f \sim \mathrm{TeV}$, as required by current EWPD (see below) if we assume the $\kappa$ eigenvalues to be order 1 . While the $\mu$ eigenvalues shall be much smaller than the $\mathrm{GeV}$. The predictions for the SM neutrino masses and the LFV contributions of the quasi-Dirac singlets $\chi$ are those of the inverse see-saw [50-52]. (See [65-70] for analyses in alternative SM extensions, including models with warped extra dimensions.) Before going through the corresponding phenomenological study, let us comment on two other a priori less natural scenarios.

\section{$2.1 \quad$ T-odd heavy singlet}

If we had chosen the T-parity of $\chi_{R}$ to be odd by defining the T-action on the fermions $\Psi_{1} \stackrel{\mathrm{T}}{\longleftrightarrow}-\Sigma_{0} \Psi_{2}, \Psi_{R} \stackrel{\mathrm{T}}{\longrightarrow}-\Psi_{R}$ and hence, the T-invariant Yukawa Lagrangian in eq. (2.7) to be $\mathcal{L}_{Y_{H}}=-\kappa f\left(\bar{\Psi}_{2} \xi+\bar{\Psi}_{1} \Sigma_{0} \Omega \xi^{\dagger} \Omega\right) \Psi_{R}+$ h.c., all new fermions would be T-odd [11]. Thus, their contribution to the mass of the SM neutrinos, once LN is broken as assumed before, would be one-loop suppressed. This appealing possibility has the drawback that the LFV Higgs decays into two charged leptons become logarithmically divergent due to the contribution of $\chi_{R}$ when exchanged in the diagrams in figure 2. (We will provide further details elsewhere.) As we are interested in setting up a predictive model at leading order

\footnotetext{
${ }^{3}$ The Higgs boson mass is also free of quadratically divergent contributions of order $\kappa^{2}$ if the $\mathrm{SO}(5)(\mathrm{RH})$ multiplets are complete and the SM singlets $\chi_{L}$ are doubled by including them in the SU(5) multiplets $\Psi_{1,2}$ in eq. (2.3). However, the Yukawa Lagrangian in eq. (2.7) also provides a large mixing between $\nu_{L}$ and (the T-even combination of) $\chi_{L}$, whose relatively large value $\sim v / 2 f$ is fixed. Hence, new mass term contributions are needed to make the lepton singlets heavier (than $\sim \sqrt{2} \kappa f$ ) and satisfy the limit on the singlet content of light neutrinos (mainly electro-weak doublets), which is bound to be $<0.03$ at $95 \%$ C.L. [62-64] (see below), without pushing $f$ too high. We will not consider this enlarged lepton content any further.
} 

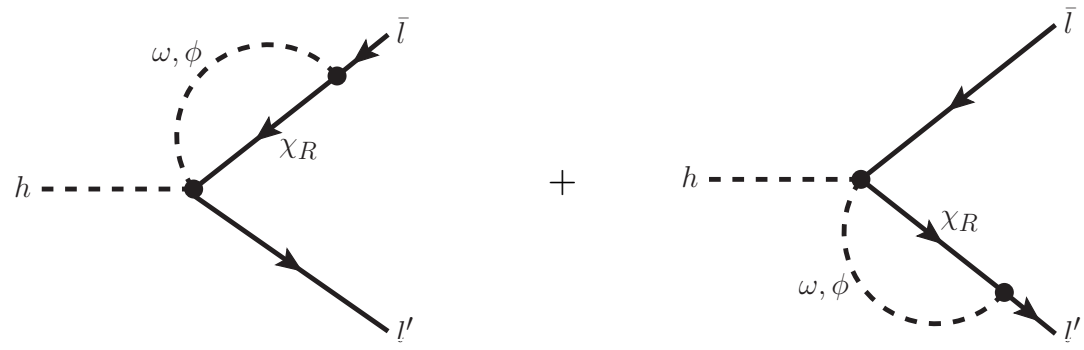

Figure 2. Higgs decay diagrams exchanging the $\mathrm{RH}$ singlet in $\mathrm{SO}(5)$ quintuplets resulting in an unmatched divergent contribution when SM charged leptons get their mass from the usual (minimal) Yukawa coupling.

at least to one loop, meaning in our case that the Higgs boson mass can get one-loop corrections at most logarithmically divergent and that LFV processes involving only SM external fields must be finite, we disregard this alternative in what follows. Nevertheless, the logarithmically divergent contribution of $\chi_{R}$ to LFV Higgs decays into charged fermions does rely on the mechanism giving masses to them. In our case, we assume that the charged leptons $\ell$ get their masses through the Yukawa Lagrangian [13, 15, 28] (summation over $x, y, z=3,4,5$ and $r, s=1,2$ is understood):

$$
\mathcal{L}_{Y}=\frac{i \lambda}{2 \sqrt{2}} f \epsilon^{x y z} \epsilon^{r s}\left[\left(\overline{\Psi_{2}^{\sigma}}\right)_{x}(\Sigma)_{r y}(\Sigma)_{s z}+\left(\overline{\Psi_{1}^{\sigma^{\prime}}} \Sigma_{0} \Omega\right)_{x}\left(\Sigma^{\prime}\right)_{r y}\left(\Sigma^{\prime}\right)_{s z}\right] \ell_{R}+\text { h.c. },
$$

where the LH leptons are included in two other incomplete $\mathrm{SU}(5)$ multiplets in fundamental representations $\underline{5}$ and $\underline{5}^{*}$, respectively:

$$
\Psi_{1}^{\sigma^{\prime}}=\left(\begin{array}{c}
\sigma^{\prime} l_{1 L} \\
0 \\
0
\end{array}\right), \quad \Psi_{2}^{\sigma}=\left(\begin{array}{c}
0 \\
0 \\
\sigma l_{2 L}
\end{array}\right)
$$

with $\sigma, \sigma^{\prime}$ scalars with the proper charges to endow $\sigma l_{2 L}$ and $\sigma^{\prime} l_{1 L}$ with the charges of the corresponding components of $\underline{5}^{*}$ and $\underline{5}$, respectively, and $\Sigma^{\prime}=\Omega \Sigma_{0} \Sigma^{\dagger} \Sigma_{0} \Omega$. Thus, the introduction of the scalars $\sigma, \sigma^{\prime}$ allows us to change the sign of the gauged $\mathrm{U}(1)$ charges in $\mathrm{SU}(5)$ for $l_{2 L}$ and $l_{1 L}$ while also giving the correct hypercharge to $\sigma l_{2 L}$ and $\sigma^{\prime} l_{1 L}$. The action under T-parity is then defined as

$$
\Psi_{1}^{\sigma^{\prime}} \stackrel{\mathrm{T}}{\longleftrightarrow} \Omega \Sigma_{0} \Psi_{2}^{\sigma}
$$

However, this particular construction does not allow to allocate $\chi_{L}$ in $\Psi_{1}^{\sigma^{\prime}}$ or $\Psi_{2}^{\sigma}$ and then, no coupling to $\ell_{R}$ can compensate for the logarithmically divergent contribution of $\chi_{R}$ in figure 2. If we wanted to insist in $\chi$ being T-odd and hence, in introducing the adequate $(h+v)^{2} \omega^{+} \overline{\chi_{L}} \ell_{R}$ and $(h+v)^{2} \Phi^{+} \overline{\chi_{L}} \ell_{R}$ couplings to compensate this $\chi_{R}$ contribution, we would have to assign $\ell_{R}$ to a larger representation, for instance, generalizing the proposal for composite Higgs models advocated in [71, 72], as we will review elsewhere. 


\section{$2.2 \quad$ See-saw of type I and II}

As mentioned when describing the NG boson content of the model at the beginning of this section, the triplet $\Phi$ has the correct SM quantum numbers to mediate the see-saw of type II. What originally brought to consider this mechanism to generate neutrino masses in the LHT [48]. However, the contribution of a non-zero vev for $\Phi^{0}$ is expected to be subleading, as we shall argue. The Yukawa couplings giving large masses to mirror leptons in eq. (2.7) fix the $\Phi$ LN to be zero and quantum corrections do not generate the see-saw operator of type II [42-47]:

$$
\mathcal{L}_{\text {see-saw }}^{I I}=y_{i j} \tilde{\tilde{l}}_{L i} \Delta l_{L j}+\text { h.c. } \rightarrow-\frac{1}{2} m_{\nu j i}^{*} \overline{\nu_{L i}^{c}} \nu_{L j}+\text { h.c., with } m_{\nu j i}^{*}=\sqrt{2} y_{i j}\left\langle\Phi^{0}\right\rangle,
$$

where $\tilde{l}_{L i}=i \sigma^{2} l_{L i}^{c}=\left(\ell_{L i}^{c}-\nu_{L i}^{c}\right)^{T}$, with $l_{L i}^{c}$ the three charge-conjugated SM lepton doublets, $i=1,2,3$, and

$$
\Delta=\left(\begin{array}{cc}
\frac{\Phi^{+}}{\sqrt{2}} & -\Phi^{++} \\
\frac{\Phi^{0}+i \Phi^{P}}{\sqrt{2}} & -\frac{\Phi^{+}}{\sqrt{2}}
\end{array}\right) .
$$

(Note that we have included a $-i \sigma^{2}$ factor on the right in the definition of $\Delta$ to take care of this factor in the definition of $\Psi$ in eq. (2.3).) This coupling not only violates LN, which must be assumed to be broken at some stage, but also T-parity because $\Phi$ is T-odd while the SM fermions are T-even. Obviously, T-parity is spontaneously broken if $\left\langle\Phi^{0}\right\rangle \neq 0$ and SM neutrinos shall get a mass once LN is broken. Nevertheless, these masses must be induced by a T-parity preserving operator, which must then involve an even number of $\Phi^{0}$ 's and be of higher dimension than the see-saw operator of type II above. As a matter of fact, an SM invariant operator must also involve at least two Higgs doublets because they must compensate for the hypercharge of the two lepton doublets. In summary, an SM and T invariant LN violating operator involving $\Phi^{0}$ is at least suppressed by a factor $\left\langle\Phi^{0}\right\rangle^{2} / f^{2}$ relative to the SM Weinberg operator [73], as we show below for the inverse see-saw, and then, it is subleading in the LHT.

In the inverse see-saw model at hand the integration out of the quasi-Dirac neutrinos $\chi$, with heavy masses given to leading order by the $3 \times 3$ mass matrix $M$, generates the corresponding Weinberg operator (see eqs. (2.7), (2.9) and (2.10) $)^{4}$

$$
\frac{1}{2}\left(\kappa f M^{-1}\right)^{*} \mu\left(\kappa f M^{-1}\right)^{\dagger}\left(\mathcal{O}_{\chi}+\mathcal{O}_{\chi}^{\prime}\right)
$$

with (also omitting family indices)

$$
\begin{aligned}
& \mathcal{O}_{\chi}=\overline{\Psi_{1}^{c}} \xi \frac{1+\Omega}{2} \xi^{T} \Psi_{1}+\overline{\Psi_{2}^{c}} \xi^{*} \frac{1+\Omega}{2} \xi^{\dagger} \Psi_{2}, \\
& \mathcal{O}_{\chi}^{\prime}=\overline{\Psi_{1}^{c}} \xi \frac{1+\Omega}{2} \xi^{\dagger} \Psi_{2}+\overline{\Psi_{2}^{c}} \xi^{*} \frac{1+\Omega}{2} \xi^{T} \Psi_{1} .
\end{aligned}
$$

\footnotetext{
${ }^{4}$ The fermionic kinetic terms properly normalized are written, for instance, in ref. [25].
} 

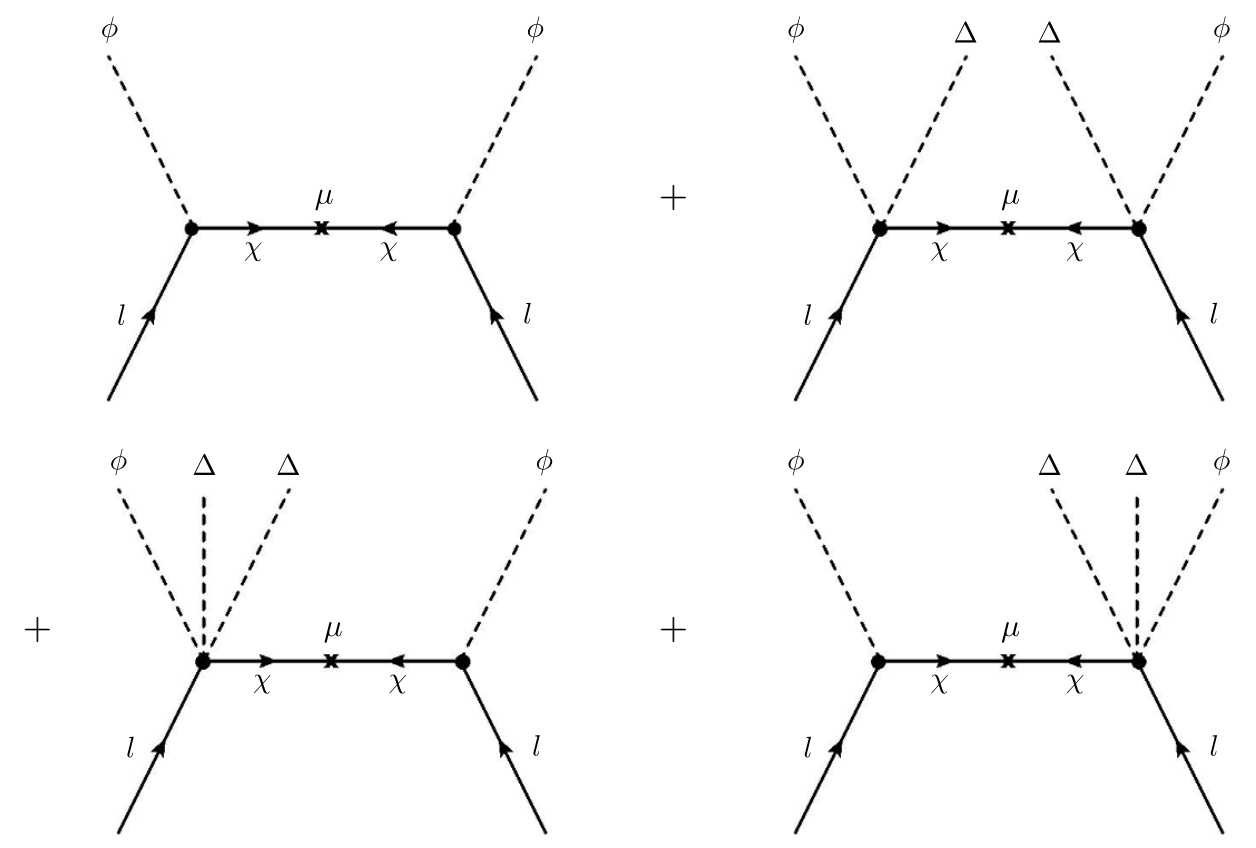

Figure 3. Diagrammatic expansion of the tree-level integration out of $\chi$, eq. (2.20).

Both $\mathcal{O}_{\chi}$ and $\mathcal{O}_{\chi}^{\prime}$ include the SM Weinberg operator and subleading contributions containing $\Phi^{0}$ (we omit subleading terms not involving $\Delta$ )

$$
\begin{aligned}
\mathcal{O}_{\chi} \supset-\frac{1}{2 f^{2}}\left(\overline{l_{L}^{c}} \tilde{\phi}^{*}\right)\left(\tilde{\phi}^{\dagger} l_{L}\right)-\frac{1}{4 f^{4}}\left[\frac{1}{2}\left(\overline{l_{L}^{c}} \Delta^{T} \phi^{*}\right)\left(\phi^{\dagger} \Delta l_{L}\right)\right. \\
\left.-\frac{1}{3}\left(\overline{l_{L}^{c}} \Delta^{T} \Delta^{*} \tilde{\phi}^{*}\right)\left(\tilde{\phi}^{\dagger} l_{L}\right)-\frac{1}{3}\left(\overline{l_{L}^{c}} \tilde{\phi}^{*}\right)\left(\tilde{\phi}^{\dagger} \Delta^{\dagger} \Delta l_{L}\right)\right]+\ldots, \\
\mathcal{O}_{\chi}^{\prime} \supset-\frac{1}{2 f^{2}}\left(\overline{l_{L}^{c}} \tilde{\phi}^{*}\right)\left(\tilde{\phi}^{\dagger} l_{L}\right)+\frac{1}{4 f^{4}}\left[\frac{1}{2}\left(\overline{l_{L}^{c}} \Delta^{T} \phi^{*}\right)\left(\phi^{\dagger} \Delta l_{L}\right)\right. \\
\left.+\frac{1}{3}\left(\overline{l_{L}^{c}} \Delta^{T} \Delta^{*} \tilde{\phi}^{*}\right)\left(\tilde{\phi}^{\dagger} l_{L}\right)+\frac{1}{3}\left(\overline{l_{L}^{c}} \tilde{\phi}^{*}\right)\left(\tilde{\phi}^{\dagger} \Delta^{\dagger} \Delta l_{L}\right)\right]+\ldots,
\end{aligned}
$$

with $\tilde{\phi}=i \sigma^{2} \phi^{*}$. (The four terms in the operator expansion can be also read from the diagrammatic tree-level integration out of $\chi$ in figure 3.) As emphasized above, the dimension 7 operators give an extra contribution to the SM neutrino masses but proportional to $\left\langle\Phi^{0}\right\rangle^{2} v^{2} / f^{3}$ and then subleading, being its ratio to the leading term $-\left\langle\Phi^{0}\right\rangle^{2} / 6 f^{2}{ }^{5}$ Thus,

\footnotetext{
${ }^{5}$ The corresponding LHT Weinberg operators preserving the full global symmetry write (see also [21])

$$
\mathcal{O}=\overline{\Psi_{1}^{c}} \Sigma \Psi_{1}+\overline{\Psi_{2}^{c}} \Sigma^{\dagger} \Psi_{2}, \quad \mathcal{O}^{\prime}=\overline{\Psi_{1}^{c}} \Psi_{2}+\overline{\Psi_{2}^{c}} \Psi_{1},
$$

where $\Sigma=\xi \Sigma_{0} \xi^{T}$ is a $5 \times 5$ symmetric tensor under $\mathrm{SU}(5), \Sigma \rightarrow V \Sigma V^{T}$, and $\Sigma_{0}$, introduced in eq. (2.5), is the singlet direction under $\mathrm{SO}(5), \Sigma_{0}=U \Sigma_{0} U^{T}$. The expansion of $\mathcal{O}$ also includes the SM Weinberg
} operator as well as the lowest order operators (of dimension 7) involving the scalar triplet $\Phi$,

$$
\begin{aligned}
\mathcal{O} \supset-\frac{1}{f^{2}}\left(\overline{l_{L}^{c}} \tilde{\phi}^{*}\right)\left(\tilde{\phi}^{\dagger} l_{L}\right)-\frac{1}{3 f^{4}}\left[\left(\overline{l_{L}^{c}} \Delta^{T} \phi^{*}\right)\left(\phi^{\dagger} \Delta l_{L}\right)\right. \\
\left.-\left(\overline{l_{L}^{c}} \Delta^{T} \Delta^{*} \tilde{\phi}^{*}\right)\left(\tilde{\phi}^{\dagger} l_{L}\right)-\left(\overline{l_{L}^{c}} \tilde{\phi}^{*}\right)\left(\tilde{\phi}^{\dagger} \Delta^{\dagger} \Delta l_{L}\right)\right]+\ldots
\end{aligned}
$$


the scalar triplet contribution to neutrino masses is expected to be very much suppressed relative to the see-saw of type I. (We shall assume in the following a vanishing $\left\langle\Phi^{0}\right\rangle$.)

\section{Inverse see-saw masses and mixings}

The inverse see-saw has been widely studied in the literature $[49-52,65,68,70]$. The light ( $l$ ) neutrino masses can be obtained diagonalizing the neutrino mass matrix in eq. (2.12) to leading order or directly from eqs. (2.18) and (2.20):

$$
\left(\mathcal{M}_{\nu}^{l}\right)_{i j}=\theta_{i k}^{*} \mu_{k l} \theta_{j l}^{*}, \quad \text { with } \quad \theta_{i k}=-i f \sin \left(\frac{v}{\sqrt{2} f}\right) \kappa_{i k} M_{k}^{-1},
$$

where we have reintroduced the family indices (summation is understood when they are repeated in a product) and assumed without lost of generality that the $\chi$ mass matrix, $M$, is diagonal and positive definite. At the same time (in the basis where the charged lepton mass matrix is diagonal)

$$
\mathcal{M}_{\nu}^{l}=U_{\mathrm{PMNS}}^{*} \mathcal{D}_{\nu}^{l} U_{\mathrm{PMNS}}^{\dagger} \text { and solving eq. (3.1), } \mu=\left(\theta^{*}\right)^{-1} U_{\mathrm{PMNS}}^{*} \mathcal{D}_{\nu}^{l} U_{\mathrm{PMNS}}^{\dagger}\left(\theta^{\dagger}\right)^{-1},
$$

where $U_{\mathrm{PMNS}}$ is the Pontecorvo-Maki-Nakagawa-Sakata mixing matrix [74-77] and $\mathcal{D}_{\nu}^{l}$ the diagonal neutrino mass matrix. Hence, for a non-singular $\theta$ matrix $\mu$ can be always adjusted to fit light neutrino masses and mixing. (We use $\mu$ to denote the Majorana matrix for $\chi_{L i}$ or any of its small entries, what should be clear by the context.) For instance, if $f>1 \mathrm{TeV}$, $\kappa=1$ and $M=10 \mathrm{TeV}, \mu \sim 0.3 \mathrm{keV}$ for a light neutrino mass of $0.1 \mathrm{eV} .^{6}$

The experimental limits on $\theta$ can be always satisfied without implementing flavor symmetries in the model but LFV constraints set stringent limits on the heavy scale as well as on the mixing between light and heavy leptons, as we review in the following.

\subsection{LFV limits}

The $\theta$ matrix elements give the mixing between light and heavy (quasi-Dirac) neutrinos, $l$ and $h$, respectively,

$$
\left(U_{\mathrm{PMNS}}\right)_{i j} \nu_{L j}^{l}=\left[\mathbf{1}_{3 \times 3}-\frac{1}{2}\left(\theta \theta^{\dagger}\right)\right]_{i j} \nu_{L j}-\theta_{i j} \chi_{L j}, \quad \chi_{L i}^{h}=\left[\mathbf{1}_{3 \times 3}-\frac{1}{2}\left(\theta^{\dagger} \theta\right)\right]_{i j} \chi_{L j}+\theta_{i j}^{\dagger} \nu_{L j},
$$

to leading order. They are constrained by lepton flavor conserving processes at tree level because they modify the SM charged and neutral currents (in standard notation) [79, 80]: ${ }^{7}$

$$
\begin{aligned}
& \mathcal{L}_{W}^{l}=\frac{g}{\sqrt{2}} \overline{\nu_{L i}^{l}} W_{i j} \gamma^{\mu} \ell_{L j} W_{\mu}^{+}+\text {h.c. }, \quad \text { with } \quad W_{i j}=\left\{U_{\mathrm{PMNS}}^{\dagger}\left[\mathbf{1}_{3 \times 3}-\frac{1}{2}\left(\theta \theta^{\dagger}\right)\right]\right\}_{i j}, \\
& \mathcal{L}_{Z}^{l}=\frac{g}{2 c_{W}} \overline{\nu_{L i}^{l}} X_{i j} \gamma^{\mu} \nu_{L j}^{l} Z_{\mu}, \quad \quad \text { with } \quad X_{i j}=\left\{U_{\mathrm{PMNS}}^{\dagger}\left[\mathbf{1}_{3 \times 3}-\left(\theta \theta^{\dagger}\right)\right] U_{\mathrm{PMNS}}\right\}_{i j}
\end{aligned}
$$

While $\mathcal{O}^{\prime}$ has no scalar couplings, and with the fermion content in eq. (2.3) it vanishes.

${ }^{6}$ Note that the small mass parameters $\mu \sim 0.3 \mathrm{keV}$ are technically natural [78], as they are the only terms in the theory breaking LN. Although in the absence of a flavor symmetry there is no dynamical explanation for the $\kappa$ and $\mu$ values fulfilling eq. (3.2).

${ }^{7}$ Charged and neutral currents are related at leading order $X_{i j}=W_{i k} W_{j k}^{*}$, with the neutral currents satisfying the positivity constraints $\left|X_{i j}\right|^{2} \leq X_{i i} X_{j j},\left|\delta_{i j}-X_{i j}\right|^{2} \leq\left(1-X_{i i}\right)\left(1-X_{j j}\right)$. The latter reduces to the Schwarz inequality $\left|\left(\theta \theta^{\dagger}\right)_{i j}\right|^{2} \leq\left(\theta \theta^{\dagger}\right)_{i i}\left(\theta \theta^{\dagger}\right)_{j j}$. All of them are automatically taken care working only with the mixing matrix elements $\theta_{i j}$. 


\begin{tabular}{|c|c|c|}
\hline \multicolumn{3}{|c|}{ EWPD $\quad$ (only one $\theta_{i i} \neq 0$, at $95 \%$ C.L. $[63]$ ) } \\
\hline$\left|\theta_{e 1}\right|<0.04$ & $\left|\theta_{\mu 2}\right|<0.03$ & $\left|\theta_{\tau 3}\right|<0.09$ \\
\hline \multicolumn{3}{|c|}{ LFV at $90 \%$ C.L. $\left(M_{k}=10 \mathrm{TeV}\right)$} \\
\hline $\operatorname{Br}(\mu \rightarrow e \gamma)<4.2 \times 10^{-13}[82]$ & $\operatorname{Br}(\tau \rightarrow e \gamma)<3.3 \times 10^{-8}$ & $\operatorname{Br}(\tau \rightarrow \mu \gamma)<4.4 \times 10^{-8}$ \\
\hline$-\theta_{e j} \theta_{\mu j}^{*} \mid<0.14 \times 10^{-4}$ & $-\theta_{e j} \theta_{\tau j}^{*} \mid<0.40 \times 10^{-2}$ & $-\theta_{\mu j} \theta_{\tau j}^{*} \mid<0.46 \times 10^{-2}$ \\
\hline
\end{tabular}

Table 1. Limits on the mixing between the SM and the heavy quasi-Dirac neutrinos from electroweak precision data (top) and from lepton flavor violating processes (bottom). The sum on the repeated index $j=1,2,3$ is understood.

More stringent are the constraints from (charged) LFV processes which proceed at one loop, as do $(g-2)_{\ell}$ and at higher order the Electric Dipole Moment of the electron $\left(\mathrm{EDM}_{e}\right) .^{8}$ Even though they are suppressed by the corresponding loop factors $1 / 16 \pi^{2}$, they can and do significantly restrict the $\theta$ matrix elements (and the heavy neutrino masses $M_{i}$ ) fixing the coupling between the SM leptons and the heavy quasi-Dirac neutrinos:

$$
\mathcal{L}_{W}^{l h}=\frac{g}{\sqrt{2}} \overline{\chi_{L i}^{h}} \theta_{i j}^{\dagger} \gamma^{\mu} \ell_{L j} W_{\mu}^{+}+\text {h.c. }, \quad \mathcal{L}_{Z}^{l h}=\frac{g}{2 c_{W}} \overline{\chi_{L i}^{h}}\left(\theta^{\dagger} U_{\mathrm{PMNS}}\right)_{i j} \gamma^{\mu} \nu_{L j}^{l} Z_{\mu}+\text { h.c. } .
$$

The Yukawa coupling in eq. (2.7) also enters in the calculation of Higgs decays, for instance,

$$
\mathcal{L}_{h}^{\nu} \supset \frac{i}{\sqrt{2}} \cos \left(\frac{v}{\sqrt{2} f}\right) \overline{\nu_{L i}} \kappa_{i j} \chi_{R j} h+\text { h.c. } \simeq-\overline{\nu_{L i}^{l}}\left(U_{\mathrm{PMNS}}^{\dagger} \theta\right)_{i j} \frac{M_{j}}{v} \chi_{R j} h+\text { h.c. },
$$

where the last equation gives the leading term in $v / f$ and $\theta_{i j}$.

In order to properly confront the LHT with experiment we should perform a global fit to EWPD and to current LFV experimental limits. This is, however, beyond the scope of this paper, in particular because there are also other one-loop contributions to the latter mediated by T-odd leptons [31, 32]. Moreover, while the amplitudes exchanging T-odd leptons are suppressed by inverse powers of $f$, the amplitudes exchanging heavy T-even neutrinos are suppressed by inverse powers of their masses $M_{k}$ and hence, their sizes can be made to vary a priori independently. We would then only derive conservative bounds, postponing a global fit to a future publication.

In the top part of table 1 we collect the limits from EWPD obtained assuming that each heavy neutrino only mixes with one light neutrino of definite flavor and that only one mixing is non-vanishing at a time [62-64]. This means that only $\theta_{i i} \neq 0$ in the basis where the charged leptons are diagonal. Assuming universality and, in particular, that the three mixings $\theta_{i i}$ are equal, their absolute value is found to be $<0.03$ at $95 \%$ C.L. [64]. Hence, eq. (3.1) implies

$$
\left|\kappa_{i i}\right|<0.17\left(\frac{M_{i}}{\mathrm{TeV}}\right)
$$

\footnotetext{
${ }^{8}$ The addition of heavy neutrinos does not modify the SM neutral currents for charged leptons at tree level and then, they remain lepton flavor conserving and universal.
} 
for $f$ larger than the TeV. Note that this effective description requires $M_{i} \lesssim 4 \pi f \sim 10 \mathrm{TeV}$ for consistency of the model. What translates into an upper bound on $\kappa_{i i}$ and in turn, into an upper bound on the mass of (T-odd) mirror leptons $\simeq \sqrt{2} \kappa f$ (see below).

LFV further restricts the mixing between light and heavy neutrinos, especially for the first two families. A solution satisfying current bounds is to assume $\theta$ diagonal, as above, banishing LFV for none has been observed up to now. However, the Yukawa coupling $\kappa$ is an arbitrary $3 \times 3$ matrix and hence, in general

$$
\kappa=V^{\dagger} \kappa^{\operatorname{diag}} Z,
$$

with $V$ and $Z$ unitary matrices and $\kappa^{\text {diag }}$ a diagonal matrix with semipositive eigenvalues. $V$ is the transformation matrix relating the mass eigenvector basis for $l_{H L}$ with the $\ell_{L}$ one [25] and $Z$ is the transformation relating the mass eigenvector basis for $l_{H R}$ with the $\chi_{R}$ one. Nevertheless, this parameterization will only matter when performing a general global fit. When performing it we shall find that for particular values of these Yukawa couplings some of the LFV observables can cancel, as found when studying the contributions of the T-odd leptons in [31, 32]. But not all of them will vanish at the same time, except in the singular case when all heavy leptons are degenerate or the heavy sector is aligned with the SM. The allowed parameter region will be then restricted by the non-vanishing observables. The size of this region and the amount of fine-tuning are determined by the most stringent bounds. However, such a phenomenological discussion is beyond the scope of this paper, as already emphasized. In order to estimate the size of these regions is sufficient to consider the most restrictive current bounds, which are obtained from the non-observation of the radiative decays $\ell \rightarrow \ell^{\prime} \gamma$ (see table 11 in [32], and ref. [81]). In table 1 we gather the corresponding limits. The contribution of the heavy (quasi-Dirac) neutrinos can be evaluated in the 't Hooft-Feynman gauge in a similar way as the contribution of the (T-odd) mirror neutrinos in [25]. (The necessary Feynman rules and loop contributions are reviewed elsewhere.)

Gauge invariance reduces the $\ell \rightarrow \ell^{\prime} \gamma$ vertex for an on-shell photon to a dipole transition,

$$
i \Gamma_{\gamma}^{\mu}\left(p_{\ell}, p_{\ell^{\prime}}\right)=i e\left[i F_{M}^{\gamma}\left(Q^{2}\right)+F_{E}^{\gamma}\left(Q^{2}\right) \gamma_{5}\right] \sigma^{\mu \nu} Q_{\nu}
$$

where $Q_{\nu}=\left(p_{\ell^{\prime}}-p_{\ell}\right)_{\nu}$. Being the decay width (neglecting $m_{\ell^{\prime}}\left(\ll m_{\ell}\right)$ )

$$
\Gamma\left(\ell \rightarrow \ell^{\prime} \gamma\right)=\frac{\alpha}{2} m_{\ell}^{3}\left(\left|F_{M}^{\gamma}\right|^{2}+\left|F_{E}^{\gamma}\right|^{2}\right)
$$

where $\alpha=e^{2} / 4 \pi$, and the form factor (defining $\alpha_{W}=\alpha / s_{W}^{2}$ )

$$
F_{M}^{\gamma}=\theta_{\ell^{\prime} j} \theta_{\ell j}^{*} \frac{\alpha_{W}}{16 \pi} \frac{m_{\ell}}{M_{W}^{2}} F_{M}^{\chi}\left(\frac{M_{W}^{2}}{M_{j}^{2}}\right),
$$

with

$$
F_{M}^{\chi}(x)=-\frac{2+5 x-x^{2}}{4(1-x)^{3}}-\frac{3 x}{2(1-x)^{4}} \ln x \quad \stackrel{x \rightarrow 0}{\longrightarrow}-\frac{1}{2},
$$


and $F_{M}^{\gamma}=-i F_{E}^{\gamma}$. While the corresponding branching ratio reads ${ }^{9}$

$$
\operatorname{Br}\left(\ell \rightarrow \ell^{\prime} \gamma\right)=\frac{3 \alpha}{2 \pi}\left|\theta_{\ell^{\prime} j} \theta_{\ell j}^{*} F_{M}^{\chi}\left(\frac{M_{W}^{2}}{M_{j}^{2}}\right)\right|^{2} .
$$

Then, in order to estimate the bounds on the mixing we can substitute $F_{M}^{\chi}$ by its limit $-1 / 2$ for $M_{j}^{2} \gg M_{W}^{2}$ (see eq. (3.12)), resulting in the bounds in table 1. If we further assume the moduli of $\theta_{\mu k}$ and $\theta_{e k}$ to be less than 0.03 (see table 1), as indicated by EWPD, they must be aligned with a precision higher than $2.4 \%$ to fulfill the LFV bound on $\mu \rightarrow e \gamma$. No similar (significant) constraint can be derived from $\tau$ decays at present.

Although it is flavor conserving, we can also compute the contribution to the muon magnetic moment $a_{\mu}=2 m_{\mu} F_{M}^{\gamma}$ (see [32] for the contribution of T-odd leptons), whose current experimental value is $a_{\mu}^{\exp }=(116592091 \pm 63) \times 10^{-11}$ [33]. With the same assumptions as above $\delta a_{\mu}^{\mathrm{T}-\text { even }}=-1.2 \times 10^{-9} \theta_{\mu k} \theta_{\mu k}^{*}$ and then, equal to $-1.1 \times 10^{-12}$ for $\theta_{\mu k} \theta_{\mu k}^{*}=(0.03)^{2}$. Which is too small (and negative) to explain a significative departure from the SM prediction, $a_{\mu}^{\mathrm{SM}}=(116591823 \pm 43) \times 10^{-11}[33]$.

Similarly to the T-odd contribution to the $\mathrm{EDM}_{e}, d_{e}=-e F_{E}^{\gamma}$, the contribution of the heavy quasi-Dirac neutrinos vanishes at one loop. A full two-loop calculation [96] is beyond the scope of this paper, although its current experimental precision $\left|d_{e}\right|<1.1 \times 10^{-29} e$-cm at $90 \%$ C.L. [97] merits it.

\section{Conclusions}

The LHT is a phenomenologically viable model with a composite Higgs. It is minimal in the sense that all other (pseudo-) NG bosons are T-odd, as there are the extra gauge bosons and almost all extra fermions, while all SM fields are T-even. This translates into less stringent constraints on their indirect effects and on their direct production because they have to be always pair-produced.

Our long-term goal is to automate the calculation of the phenomenological predictions of a definite LHT model which can be confronted to experiment, as the minimal supersymmetric scenarios, and guide collider searches. This means fixing the minimal fermion content that makes the experimentally most restrictive processes one-loop finite while keeping the Higgs boson mass free from quadratic divergences. This concerns the quark as well as the lepton sector, and in this latter case the charged LFV processes which are the most stringently constrained. The contributions of the T-odd (heavy) leptons in the standard construction which are necessary to make the Higgs decays finite are calculated in refs. [31, 32]. In order to make the Higgs boson mass free of quadratic divergences one must also include the SM singlets in the $\mathrm{RH} \mathrm{SO}(5)$ quintuplets. The contributions of these heavy quasi-Dirac neutrinos to charged LFV transitions are reviewed elsewhere. In this

\footnotetext{
${ }^{9} \mathrm{~A}$ lot of attention has been payed to this process in the past [84-87] due to the stringent experimental bound on $\mu \rightarrow e \gamma$. The contribution of the heavy (quasi-Dirac) neutrinos involves the couplings in eqs. (3.4) and (3.5) as well as the couplings accounting for the Goldstone boson exchange. This has been calculated quite a few times in the past [88-95], together with other LFV transitions.
} 
paper we point out that such a working model can also accommodate neutrino masses and mixings as these heavy neutrinos allow to implement the inverse see-saw mechanism in a natural way.

If they are chosen to be T-even, they mix at tree level with the SM neutrinos, giving rise to a rich phenomenology which has attracted a lot of attention in the past [49-52, 98105]. In the LHT, however, the parameters describing this mixing are common to other sectors of the theory and this is then further constrained by the corresponding experimental observables, in particular, by EWPD and LHC production limits [106, 107]. This inverse see-saw mechanism of type I does not need to break T-parity, in contrast with the see-saw of type II induced by a non-vanishing vev of the neutral component $\left\langle\Phi^{0}\right\rangle$ of the pseudo-NG scalar triplet of hypecharge -1 present in the model. Moreover, the induced contribution in this latter case is higher order in the LHT Weinberg operator expansion and hence, further suppressed. In any case LN must be explicitly broken. A breaking which we assume to be small and deferred to the heavy LH SU(5) singlet counterpart, $\chi_{L}$, of the SM RH singlets, $\chi_{R}$, that live in $\mathrm{SO}(5)$ quintuplets. (If alternatively $\chi_{R}$ is chosen to be T-odd, the minimal coupling giving masses to the SM charged leptons has to be generalized to maintain the LFV Higgs decay into fermion pairs one-loop finite.)

As already emphasized, current experimental limits on the allowed departure from the SM predictions can be easily accommodated by the relatively large number of parameters fixing the LHT. Further fine tuning in the neutrino sector is only necessary for the LFV mixing, which has to be typically adjusted to $1 \%$ for $\mu$ to $e$ transitions. Nevertheless, the expected range of variation of the LHT parameters makes quite interesting future searches at the LHC. In the inverse see-saw mechanism the observed neutrino masses and mixings are uncorrelated, in the absence of a flavor symmetry, with the masses of the heavy quasiDirac neutrinos and their mixing with the light sector. ${ }^{10}$ In fact, the small LN violating masses $\mu$ for $\chi_{L}$ in eq. (2.10) can be adjusted to reproduce the light neutrino masses and mixings for any (non-singular) value of the heavy-light mixing (see eq. (3.2)). Quasi-Dirac neutrino masses and mixings are only bounded on the other hand by their direct production limit, which for $M$ is currently of the order of $M_{W}$ [113], ${ }^{11}$ and by the non-observation of any significant departure from the SM predictions in the leptonic sector. The common dependence on the Yukawa coupling $\kappa$ in eq. (2.7) of the mixing $\theta \simeq(v / \sqrt{2}) \kappa M^{-1}$ between the light and heavy neutrinos and of the (T-odd) mirror lepton masses $m_{\ell H} \simeq \sqrt{2} \kappa f$ delimits the $M-m_{\ell H}$ region allowed by the bound on $\theta<0.03$. Region, which is further restricted by the non-observation of heavy lepton production [107]. In figure 4 we draw these regions for $f=1.5$ and $1.9 \mathrm{TeV}$, red and black lines, respectively. In both cases quasi-

\footnotetext{
${ }^{10}$ Flavor symmetries based on $A_{4}$ or $S_{3}$ (see for instance [108-112]) could be implemented to predict the observed pattern of lepton masses and mixing angles preventing at the same time large LFV transitions. However, this goes beyond the scope of this article and it is postponed to future work.

${ }^{11}$ Quasi-Dirac neutrinos are mainly produced by the exchange of $W^{ \pm}, Z$ and $h$ at the LHC (see eqs. (3.5) and (3.6)) [103]. But these amplitudes are proportional to the heavy neutrino mixing with the SM neutrinos and it must fixed to its current upper bound of 0.03 to maximize the direct production lower bound on $M$. Besides, $\mathrm{LN}$ is practically conserved and the corresponding backgrounds are larger than in the case of heavy Majorana neutrinos. The most significant final states turn out to be three charged leptons plus missing energy [98-101], and the expected reach for $M$ of the order of $300 \mathrm{GeV}$ at the HL-LHC [105].
} 


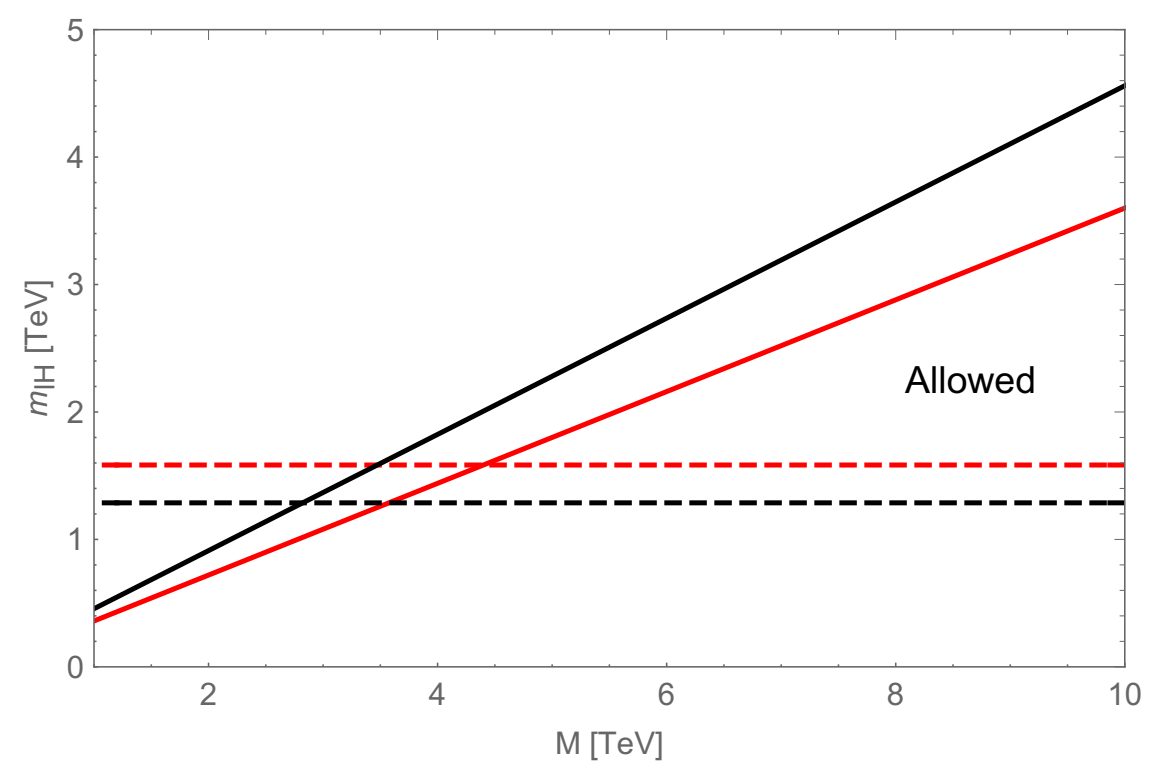

Figure 4. Allowed mass region for the mirror lepton mass $m_{\ell H}$ versus the quasi-Dirac neutrino mass $M$ for different values of the next physics scale $f$. Solid lines are fixed by the upper bound of 0.03 on the mixing between SM and heavy neutrinos for $f=1.5 \mathrm{TeV}$ (red) and $1.9 \mathrm{TeV}$ (black). While dashed lines delimit the regions excluded by the non-observation of mirror leptons.

Dirac neutrino masses below few $\mathrm{TeV}$ are excluded. It must be emphasized, however, that the $m_{\ell H}$ production limit depends on $f$ dramatically because pair production of new vectorlike leptons decaying into a SM lepton and the lightest T-odd boson (missing energy) at the LHC is very much suppressed for $f>2 \mathrm{TeV}$ [107], then drastically relaxing the lower bound on $M$. The limit from neutrino mixing will improve with a more precise determination of the constraints from EWPD while the improvement of the bound on lepton pair-production will mainly require a higher colliding energy. Both will cut down the allowed mass region in the LHT as a function of the new physics scale $f$, mainly fixed by the non-observation of new (T-odd) gauge bosons. More stringent limits on $f$ can be also derived from mirror quark production but as a function of their own Yukawa couplings [107].

\section{Acknowledgments}

We thank previous work with Ll. Ametller, P. Talavera and R. Vega-Morales and ongoing collaboration with T. Hahn, and useful comments by J. Hubisz and I. Low. This work has been supported in part by the Ministry of Science, Innovation and Universities, under grant numbers FPA2016-78220-C3-1,2,3-P (fondos FEDER), and by the Junta de Andalucía grants FQM 101 and SOMM17/6104/UGR.

Open Access. This article is distributed under the terms of the Creative Commons Attribution License (CC-BY 4.0), which permits any use, distribution and reproduction in any medium, provided the original author(s) and source are credited. 


\section{References}

[1] CMS collaboration, Observation of a new boson at a mass of $125 \mathrm{GeV}$ with the CMS experiment at the LHC, Phys. Lett. B 716 (2012) 30 [arXiv:1207.7235] [INSPIRE].

[2] ATLAS collaboration, Observation of a new particle in the search for the standard model Higgs boson with the ATLAS detector at the LHC, Phys. Lett. B 716 (2012) 1 [arXiv: 1207.7214] [INSPIRE].

[3] P. Savard, Overview of the ATLAS experiment, talk given at the XXIX International Symposium on Lepton Photon Interactions at High Energies (LP2019), August 5-10, Toronto, Canada (2019).

[4] P. McBride, Overview of the CMS experiment, talk given at the XXIX International Symposium on Lepton Photon Interactions at High Energies (LP2019), August 5-10, Toronto, Canada (2019).

[5] D.B. Kaplan and H. Georgi, $\mathrm{SU}(2) \times \mathrm{U}(1)$ breaking by vacuum misalignment, Phys. Lett. B 136 (1984) 183

[6] M.J. Dugan, H. Georgi and D.B. Kaplan, Anatomy of a composite Higgs model, Nucl. Phys. B 254 (1985) 299 [INSPIRE].

[7] K. Agashe, R. Contino and A. Pomarol, The minimal composite Higgs model, Nucl. Phys. B 719 (2005) 165 [hep-ph/0412089] [INSPIRE].

[8] N. Arkani-Hamed, A.G. Cohen, E. Katz and A.E. Nelson, The littlest Higgs, JHEP 07 (2002) 034 [hep-ph/0206021] [inSPIRE].

[9] H.-C. Cheng and I. Low, TeV symmetry and the little hierarchy problem, JHEP 09 (2003) 051 [hep-ph/0308199] [INSPIRE].

[10] H.-C. Cheng and I. Low, Little hierarchy, little Higgses and a little symmetry, JHEP 08 (2004) 061 [hep-ph/0405243] [INSPIRE].

[11] I. Low, T parity and the littlest Higgs, JHEP 10 (2004) 067 [hep-ph/0409025] [INSPIRE].

[12] H.-C. Cheng, I. Low and L.-T. Wang, Top partners in little Higgs theories with T-parity, Phys. Rev. D 74 (2006) 055001 [hep-ph/0510225] [INSPIRE].

[13] J. Hubisz and P. Meade, Phenomenology of the littlest Higgs with T-parity, Phys. Rev. D 71 (2005) 035016 [hep-ph/0411264] [INSPIRE].

[14] J. Hubisz, S.J. Lee and G. Paz, The flavor of a little Higgs with T-parity, JHEP 06 (2006) 041 [hep-ph/0512169] [INSPIRE].

[15] C.-R. Chen, K. Tobe and C.P. Yuan, Higgs boson production and decay in little Higgs models with T-parity, Phys. Lett. B 640 (2006) 263 [hep-ph/0602211] [INSPIRE].

[16] M. Blanke et al., Particle-antiparticle mixing, $\epsilon_{K}, \Delta \Gamma_{q}, A_{S L}^{q}, A_{C P}\left(B_{d} \rightarrow \psi K_{S}\right)$, $A_{C P}\left(B_{s} \rightarrow \psi \phi\right)$ and $B \rightarrow X(s, d \gamma)$ in the littlest Higgs model with T-parity, JHEP 12 (2006) 003 [hep-ph/0605214] [INSPIRE].

[17] A.J. Buras, A. Poschenrieder, S. Uhlig and W.A. Bardeen, Rare $K$ and $B$ decays in the littlest Higgs model without $T^{-}$parity, JHEP 11 (2006) 062 [hep-ph/0607189] [INSPIRE].

[18] A. Belyaev, C.-R. Chen, K. Tobe and C.P. Yuan, Phenomenology of littlest Higgs model with $T^{-}$parity: including effects of $T^{-}$odd fermions, Phys. Rev. D 74 (2006) 115020 [hep-ph/0609179] [INSPIRE].

[19] M. Blanke et al., Rare and CP-violating $K$ and $B$ decays in the littlest Higgs model with $T^{-}$ parity, JHEP 01 (2007) 066 [hep-ph/0610298] [INSPIRE]. 
[20] C.T. Hill and R.J. Hill, Topological physics of little Higgs bosons, Phys. Rev. D 75 (2007) 115009 [hep-ph/0701044] [INSPIRE].

[21] M. Blanke et al., Charged lepton flavour violation and $(g-2)_{\mu}$ in the littlest Higgs model with T-parity: a clear distinction from supersymmetry, JHEP 05 (2007) 013 [hep-ph/0702136] [INSPIRE].

[22] C.T. Hill and R.J. Hill, $T^{-}$parity violation by anomalies, Phys. Rev. D 76 (2007) 115014 [arXiv: 0705. 0697] [INSPIRE].

[23] X.-F. Han, L. Wang and J.M. Yang, Higgs and Z-boson FCNC decays correlated with B-meson decays in littlest Higgs model with T-parity, Phys. Rev. D 78 (2008) 075017 [arXiv: 0807.4480] [INSPIRE].

[24] T. Goto, Y. Okada and Y. Yamamoto, Ultraviolet divergences of flavor changing amplitudes in the littlest Higgs model with T-parity, Phys. Lett. B 670 (2009) 378 [arXiv:0809.4753] [INSPIRE].

[25] F. del Aguila, J.I. Illana and M.D. Jenkins, Precise limits from lepton flavour violating processes on the Littlest Higgs model with T-parity, JHEP 01 (2009) 080 [arXiv:0811.2891] [INSPIRE].

[26] M. Blanke et al., FCNC processes in the littlest Higgs model with T-parity: a 2009 Look, Acta Phys. Polon. B 41 (2010) 657 [arXiv:0906.5454] [InSPIRE].

[27] F. del Aguila, J.I. Illana and M.D. Jenkins, Muon to electron conversion in the Littlest Higgs model with T-parity, JHEP 09 (2010) 040 [arXiv: 1006.5914] [INSPIRE].

[28] T. Goto, Y. Okada and Y. Yamamoto, Tau and muon lepton flavor violations in the littlest Higgs model with T-parity, Phys. Rev. D 83 (2011) 053011 [arXiv:1012.4385] [INSPIRE].

[29] H.-S. Hou, H. Sun and Y.-J. Zhou, Flavor changing top quark decay and bottom-strange production in the littlest Higgs model with T-parity, Commun. Theor. Phys. 59 (2013) 443 [arXiv:1210.3904] [INSPIRE].

[30] B. Yang, J. Han and N. Liu, Lepton flavor violating Higgs boson decay $h \rightarrow \mu \tau$ in the littlest Higgs model with T parity, Phys. Rev. D 95 (2017) 035010 [arXiv:1605.09248] [InSPIRE].

[31] F. del Aguila et al., Lepton flavor changing Higgs decays in the littlest Higgs model with T-parity, JHEP 08 (2017) 028 [Erratum ibid. 02 (2019) 047] [arXiv:1705.08827] [INSPIRE].

[32] F. del Aguila et al., The full lepton flavor of the littlest Higgs model with T-parity, JHEP 07 (2019) 154 [arXiv: 1901.07058] [INSPIRE].

[33] Particle Data Group collaboration, Review of particle physics, Phys. Rev. D 98 (2018) 030001 [INSPIRE].

[34] R.N. Mohapatra, Mechanism for understanding small neutrino mass in superstring theories, Phys. Rev. Lett. 56 (1986) 561 [INSPIRE].

[35] R.N. Mohapatra and J.W.F. Valle, Neutrino mass and baryon number nonconservation in superstring models, Phys. Rev. D 34 (1986) 1642 [INSPIRE].

[36] J. Bernabeu et al., Lepton flavor nonconservation at high-energies in a superstring inspired standard model, Phys. Lett. B 187 (1987) 303 [INSPIRE].

[37] I. Esteban et al., Global analysis of three-flavour neutrino oscillations: synergies and tensions in the determination of $\theta_{23}, \delta_{\mathrm{CP}}$ and the mass ordering, JHEP 01 (2019) 106 [arXiv: 1811.05487] [INSPIRE].

[38] P. Minkowski, $\mu \rightarrow$ er at a rate of one out of $10^{9}$ muon decays?, Phys. Lett. B 67 (1977) 421. 
[39] M. Gell-Mann, P. Ramond and R. Slansky, Complex spinors and unified theories, Conf. Proc. C 790927 (1979) 315 [arXiv: 1306.4669] [InSPIRE].

[40] T. Yanagida, Horizontal gauge symmetry and masses of neutrinos, Conf. Proc. C $\mathbf{7 9 0 2 1 3 1}$ (1979) 95 [INSPIRE].

[41] R.N. Mohapatra and G. Senjanović, Neutrino mass and spontaneous parity nonconservation, Phys. Rev. Lett. 44 (1980) 912 [INSPIRE].

[42] J. Schechter and J.W.F. Valle, Neutrino masses in $\mathrm{SU}(2) \times \mathrm{U}(1)$ theories, Phys. Rev. D 22 (1980) 2227 [INSPIRE].

[43] M. Magg and C. Wetterich, Neutrino mass problem and gauge hierarchy, Phys. Lett. B 94 (1980) 61.

[44] T.P. Cheng and L.-F. Li, Neutrino masses, mixings and oscillations in $\mathrm{SU}(2) \times \mathrm{U}(1)$ models of electroweak interactions, Phys. Rev. D 22 (1980) 2860 [INSPIRE].

[45] G.B. Gelmini and M. Roncadelli, Left-handed neutrino mass scale and spontaneously broken lepton number, Phys. Lett. B 99 (1981) 411.

[46] G. Lazarides, Q. Shafi and C. Wetterich, Proton lifetime and Fermion masses in an $\mathrm{SO}(10)$ model, Nucl. Phys. B 181 (1981) 287 [INSPIRE].

[47] R.N. Mohapatra and G. Senjanović, Neutrino masses and mixings in gauge models with spontaneous parity violation, Phys. Rev. D 23 (1981) 165 [INSPIRE].

[48] T. Han, H.E. Logan, B. Mukhopadhyaya and R. Srikanth, Neutrino masses and lepton-number violation in the littlest Higgs scenario, Phys. Rev. D 72 (2005) 053007 [hep-ph/0505260] [INSPIRE].

[49] A. Abada, C. Biggio, F. Bonnet, M.B. Gavela and T. Hambye, Low energy effects of neutrino masses, JHEP 12 (2007) 061 [arXiv:0707.4058] [INSPIRE].

[50] E. Arganda, M.J. Herrero, X. Marcano and C. Weiland, Imprints of massive inverse seesaw model neutrinos in lepton flavor violating Higgs boson decays, Phys. Rev. D 91 (2015) 015001 [arXiv: 1405.4300] [INSPIRE].

[51] V. De Romeri, M.J. Herrero, X. Marcano and F. Scarcella, Lepton flavor violating Z decays: a promising window to low scale seesaw neutrinos, Phys. Rev. D 95 (2017) 075028 [arXiv: 1607.05257] [INSPIRE].

[52] P. Ballett, T. Boschi and S. Pascoli, Heavy neutral leptons from low-scale seesaws at the DUNE near detector, arXiv:1905.00284 [INSPIRE].

[53] M. Schmaltz and D. Tucker-Smith, Little Higgs review, Ann. Rev. Nucl. Part. Sci. 55 (2005) 229 [hep-ph/0502182] [INSPIRE].

[54] M. Perelstein, Little Higgs models and their phenomenology, Prog. Part. Nucl. Phys. 58 (2007) 247 [hep-ph/0512128] [INSPIRE].

[55] G. Panico and A. Wulzer, The composite Nambu-Goldstone Higgs, Lecture Notes in Physics volume 913, Springer, Germany (2016), arXiv:1506.01961.

[56] D. Pappadopulo and A. Vichi, T-parity, its problems and their solution, JHEP 03 (2011) 072 [arXiv: 1007.4807] [INSPIRE].

[57] R. Balkin, G. Perez and A. Weiler, Little composite dark matter, Eur. Phys. J. C 78 (2018) 104 [arXiv: 1707.09980$]$ [INSPIRE].

[58] M. Perelstein, M.E. Peskin and A. Pierce, Top quarks and electroweak symmetry breaking in little Higgs models, Phys. Rev. D 69 (2004) 075002 [hep-ph/0310039] [INSPIRE]. 
[59] T. Han, H.E. Logan and L.-T. Wang, Smoking-gun signatures of little Higgs models, JHEP 01 (2006) 099 [hep-ph/0506313] [INSPIRE].

[60] S.R. Coleman and E.J. Weinberg, Radiative corrections as the origin of spontaneous symmetry breaking, Phys. Rev. D 7 (1973) 1888 [INSPIRE].

[61] S. Ferrara, C. Kounnas and F. Zwirner, Mass formulae and natural hierarchy in string effective supergravities, Nucl. Phys. B 429 (1994) 589 [Erratum ibid. B 433 (1995) 255] [hep-th/9405188] [INSPIRE].

[62] F. del Aguila, J. de Blas and M. Pérez-Victoria, Effects of new leptons in electroweak precision data, Phys. Rev. D 78 (2008) 013010 [arXiv:0803.4008] [INSPIRE].

[63] J. de Blas, Electroweak limits on physics beyond the Standard Model, EPJ Web Conf. 60 (2013) 19008 [arXiv: 1307.6173] [INSPIRE].

[64] J. de Blas, Effective Lagrangian description of physics beyond the standard model and electroweak precision tests, Ph.D. Thesis, University of Granada, Granada, Spain (2010).

[65] M. Malinsky, J.C. Romao and J.W.F. Valle, Novel supersymmetric $\mathrm{SO}(10)$ seesaw mechanism, Phys. Rev. Lett. 95 (2005) 161801 [hep-ph/0506296] [INSPIRE].

[66] K. Agashe, P. Du and S. Hong, LHC signals for singlet neutrinos from a natural warped seesaw mechanism. I, Phys. Rev. D 97 (2018) 075032 [arXiv:1612.04810] [InSPIRE].

[67] K. Agashe, P. Du and S. Hong, LHC signals for singlet neutrinos from a natural warped seesaw mechanism. II, Phys. Rev. D 97 (2018) 075033 [arXiv:1703.07763] [InSPIRE].

[68] V. De Romeri, K.M. Patel and J.W.F. Valle, Inverse seesaw mechanism with compact supersymmetry: Enhanced naturalness and light superpartners, Phys. Rev. D 98 (2018) 075014 [arXiv: 1808.01453] [INSPIRE].

[69] K. Agashe et al., Natural seesaw and leptogenesis from hybrid of high-scale Type I and TeV-scale inverse, JHEP 04 (2019) 029 [arXiv: 1812.08204] [INSPIRE].

[70] A.E. Cárcamo Hernández and S.F. King, Littlest inverse seesaw model, arXiv:1903.02565 [INSPIRE].

[71] R. Contino, L. Da Rold and A. Pomarol, Light custodians in natural composite Higgs models, Phys. Rev. D 75 (2007) 055014 [hep-ph/0612048] [INSPIRE].

[72] C. Csáki, A. Falkowski and A. Weiler, The flavor of the composite pseudo-Goldstone Higgs, JHEP 09 (2008) 008 [arXiv:0804.1954] [INSPIRE].

[73] S. Weinberg, Baryon and Lepton nonconserving processes, Phys. Rev. Lett. 43 (1979) 1566 [INSPIRE].

[74] B. Pontecorvo, Mesonium and anti-mesonium, Sov. Phys. JETP 6 (1957) 429 [Zh. Eksp. Teor. Fiz. 33 (1957) 549] [INSPIRE].

[75] B. Pontecorvo, Inverse beta processes and nonconservation of lepton charge, Sov. Phys. JETP 7 (1958) 172 [Zh. Eksp. Teor. Fiz. 34 (1957) 247] [INSPIRE].

[76] B. Pontecorvo, Neutrino experiments and the problem of conservation of leptonic charge, Sov. Phys. JETP 26 (1968) 984 [Zh. Eksp. Teor. Fiz. 53 (1967) 1717] [InSPIRE].

[77] Z. Maki, M. Nakagawa and S. Sakata, Remarks on the unified model of elementary particles, Prog. Theor. Phys. 28 (1962) 870 [inSPIRE].

[78] G. 't Hooft, Naturalness, chiral symmetry, and spontaneous chiral symmetry breaking, NATO Sci. Ser. B 59 (1980) 135.

[79] F. del Aguila, M. Pérez-Victoria and J. Santiago, Effective description of quark mixing, Phys. Lett. B 492 (2000) 98 [hep-ph/0007160] [INSPIRE]. 
[80] F. del Aguila, M. Pérez-Victoria and J. Santiago, Observable contributions of new exotic quarks to quark mixing, JHEP 09 (2000) 011 [hep-ph/0007316] [INSPIRE].

[81] A. Baldini et al., A submission to the 2020 update of the European Strategy for Particle Physics on behalf of the COMET, MEG, Mu2e and Muße collaborations, arXiv: 1812.06540 [INSPIRE].

[82] MEG collaboration, New constraint on the existence of the $\mu^{+} \rightarrow e^{+} \gamma$ decay, Phys. Rev. Lett. 110 (2013) 201801 [arXiv: 1303. 0754] [INSPIRE].

[83] BABAR collaboration, Searches for lepton flavor violation in the decays $\tau^{ \pm} \rightarrow e^{ \pm} \gamma$ and $\tau^{ \pm} \rightarrow \mu^{ \pm} \gamma$, Phys. Rev. Lett. 104 (2010) 021802 [arXiv:0908.2381] [INSPIRE].

[84] F. del Aguila and M.J. Bowick, Suppression of lepton number violation mediated by $\Delta I=0$ mass fermions, Phys. Lett. B 119 (1982) 144.

[85] J. Hisano, T. Moroi, K. Tobe and M. Yamaguchi, Lepton flavor violation via right-handed neutrino Yukawa couplings in supersymmetric standard model, Phys. Rev. D 53 (1996) 2442 [hep-ph/9510309] [INSPIRE].

[86] J.I. Illana and M. Masip, Lepton flavor violation in $Z$ and lepton decays in supersymmetric models, Phys. Rev. D 67 (2003) 035004 [hep-ph/0207328] [INSPIRE].

[87] E. Arganda and M.J. Herrero, Testing supersymmetry with lepton flavor violating tau and mu decays, Phys. Rev. D 73 (2006) 055003 [hep-ph/0510405] [INSPIRE].

[88] J.D. Bjorken, K.D. Lane and S. Weinberg, The decay $\mu \rightarrow e+\gamma$ in models with neutral heavy leptons, Phys. Rev. D 16 (1977) 1474 [inSPIRE].

[89] T.-P. Cheng and L.-F. Li, Muon number nonconservation effects in a gauge theory with $V A$ currents and heavy neutral leptons, Phys. Rev. D 16 (1977) 1425 [inSPIRE].

[90] C.S. Lim and T. Inami, Lepton flavor nonconservation and the mass generation mechanism for neutrinos, Prog. Theor. Phys. 67 (1982) 1569 [InSPIRE].

[91] J.D. Vergados, The neutrino mass and family, lepton and baryon nonconservation in gauge theories, Phys. Rept. 133 (1986) 1 [INSPIRE].

[92] M.C. Gonzalez-Garcia and J.W.F. Valle, Enhanced lepton flavor violation with massless neutrinos: a study of muon and $\tau$ decays, Mod. Phys. Lett. A 7 (1992) 477 [InSPIRE].

[93] A. Ilakovac and A. Pilaftsis, Flavor violating charged lepton decays in seesaw-type models, Nucl. Phys. B 437 (1995) 491 [hep-ph/9403398] [INSPIRE].

[94] D. Tommasini, G. Barenboim, J. Bernabeu and C. Jarlskog, Nondecoupling of heavy neutrinos and lepton flavor violation, Nucl. Phys. B 444 (1995) 451 [hep-ph/9503228] [INSPIRE].

[95] J.I. Illana and T. Riemann, Charged lepton flavor violation from massive neutrinos in $Z$ decays, Phys. Rev. D 63 (2001) 053004 [hep-ph/0010193] [INSPIRE].

[96] S.M. Barr and A. Zee, Electric dipole moment of the electron and of the neutron, Phys. Rev. Lett. 65 (1990) 21 [Erratum ibid. 65 (1990) 2920] [INSPIRE].

[97] ACME collaboration, Improved limit on the electric dipole moment of the electron, Nature 562 (2018) 355 [INSPIRE].

[98] F. del Aguila, J.A. Aguilar-Saavedra and R. Pittau, Heavy neutrino signals at large hadron colliders, JHEP 10 (2007) 047 [hep-ph/0703261] [INSPIRE].

[99] F. del Aguila and J.A. Aguilar-Saavedra, Electroweak scale seesaw and heavy Dirac neutrino signals at LHC, Phys. Lett. B 672 (2009) 158 [arXiv:0809.2096] [InSPIRE]. 
[100] A. Das, P.S. Bhupal Dev and N. Okada, Direct bounds on electroweak scale pseudo-Dirac neutrinos from $\sqrt{s}=8 \mathrm{TeV}$ LHC data, Phys. Lett. B 735 (2014) 364 [arXiv:1405.0177] [INSPIRE].

[101] C.O. Dib, C.S. Kim and K. Wang, Signatures of Dirac and Majorana sterile neutrinos in trilepton events at the LHC, Phys. Rev. D 95 (2017) 115020 [arXiv:1703.01934] [InSPIRE].

[102] A. Das, P.S.B. Dev and C.S. Kim, Constraining sterile neutrinos from precision Higgs data, Phys. Rev. D 95 (2017) 115013 [arXiv:1704.00880] [InSPIRE].

[103] R. Ruiz, M. Spannowsky and P. Waite, Heavy neutrinos from gluon fusion, Phys. Rev. D 96 (2017) 055042 [arXiv: 1706. 02298] [InSPIRE].

[104] Y. Cai, T. Han, T. Li and R. Ruiz, Lepton number violation: seesaw models and their collider tests, Front. Phys. 6 (2018) 40 [arXiv:1711.02180].

[105] S. Pascoli, R. Ruiz and C. Weiland, Heavy neutrinos with dynamic jet vetoes: multilepton searches at $\sqrt{s}=14,27$ and 100 TeV, JHEP 06 (2019) 049 [arXiv:1812.08750] [INSPIRE].

[106] J. Reuter, M. Tonini and M. de Vries, Littlest Higgs with T-parity: Status and Prospects, JHEP 02 (2014) 053 [arXiv: 1310.2918] [INSPIRE].

[107] D. Dercks, G. Moortgat-Pick, J. Reuter and S.Y. Shim, The fate of the littlest Higgs model with T-parity under 13 TeV LHC data, JHEP 05 (2018) 049 [arXiv:1801.06499] [INSPIRE].

[108] C. Csáki, C. Delaunay, C. Grojean and Y. Grossman, A model of lepton masses from a warped extra dimension, JHEP 10 (2008) 055 [arXiv:0806.0356] [INSPIRE].

[109] M. Hirsch, S. Morisi and J.W.F. Valle, A4-based tri-bimaximal mixing within inverse and linear seesaw schemes, Phys. Lett. B 679 (2009) 454 [arXiv:0905.3056] [INSPIRE].

[110] F. del Aguila, A. Carmona and J. Santiago, Neutrino masses from an A4 symmetry in holographic composite Higgs models, JHEP 08 (2010) 127 [arXiv:1001.5151] [INSPIRE].

[111] G. Altarelli and F. Feruglio, Discrete flavor symmetries and models of neutrino mixing, Rev. Mod. Phys. 82 (2010) 2701 [arXiv: 1002.0211] [InSPIRE].

[112] E. Ma and R. Srivastava, Dirac or inverse seesaw neutrino masses with B - L gauge symmetry and $S_{3}$ flavor symmetry, Phys. Lett. B 741 (2015) 217 [arXiv:1411.5042] [INSPIRE].

[113] CMS collaboration, Search for heavy neutral leptons in events with three charged leptons in proton-proton collisions at $\sqrt{s}=13$ TeV, Phys. Rev. Lett. 120 (2018) 221801 [arXiv: 1802.02965] [INSPIRE]. 